\title{
Applicability of Modulation Schemes for WDM High Speed Networks using Hybrid Optical Amplifiers
}

\author{
Shakshi, Himanshi Saini
}

\begin{abstract}
High speed networks face several challenges in order to meet desired Quality of Service (QOS). In order to increase network speed with significant reduction in Bit Error Rate (BER), new design techniques have to be deployed in high speed networks. In this paper, basics of high speed networks along with Dense Wavelength Division Multiplexing(DWDM) network issues and challenges have been discussed. Modulation schemes and amplifier configurations are also summarized. Investigations on applicability of modulation schemes for DWDM network architecture have been performed with various hybrid optical amplifier configurations. The link configuration consists of 56 channels at speed of 15Gbps.It is observed that Non Return to Zero (NRZ) scheme gives better performance in terms of Quality-factor $(Q-f)$ and BER. The network parameters are further improved using various hybrid optical amplifier configurations. It is observed that NRZ scheme with Erbium Doped Fiber Amplifier (EDFA) hybrid amplifier configuration has improved the quality factorof system as compared to other hybrid amplifier configurations.
\end{abstract}

Index Terms:Amplifier, Bit Error Rate (BER), Modulation,Multiplexing, Quality-factor $(Q-f)$.

\section{INTRODUCTION}

Optical fiber transmission is the effective way of communication in which light pulse is used to transfer data from one place to another place with the help of optical fiber cables. Fiber optic networks fulfill the demands of modern communication system. Optical system has several advantages like high bit rate transfer, low losses, can support over long distance communication. Due to the ever increasing demand for higher data rates and faster communication, the optical fiber communication systems proved that it is the best solution. The number of channels used for data transfer can be increased with the increase in the data rate per channel. And this can be done by Wavelength Division Multiplexing (WDM) and DWDM. In DWDM technique, multiple signals of different wavelengths are combined and transmitted over same fiber. This results in full duplex transmission and reconfigurable networking. By use of these techniques, spectral utilization can be improved which enhances the overall capacity of communication system.

The model of an optical communication system consist

Revised Manuscript Received on July 06, 2019.

Shakshi, ECE Deartment, Deenbandhu Chhotu Ram University of Science and Technology, Murthal, Sonipat, Haryana, India

Himanshi Saini, ECE Deartment, Deenbandhu Chhotu Ram University of Science and Technology, Murthal, Sonipat, Haryana, India an optical transmitter, where the information is being modulated over a carrier wave by using some modulation format, an optical fiber as the transmission medium and at optical receiver original information signal is being reconstructed, as shown in Fig. 1. The basic role of the transmitter is to convert the information from electrical to optical domain with the help of an electro-optic modulator and the receiver performs the reverse operations.

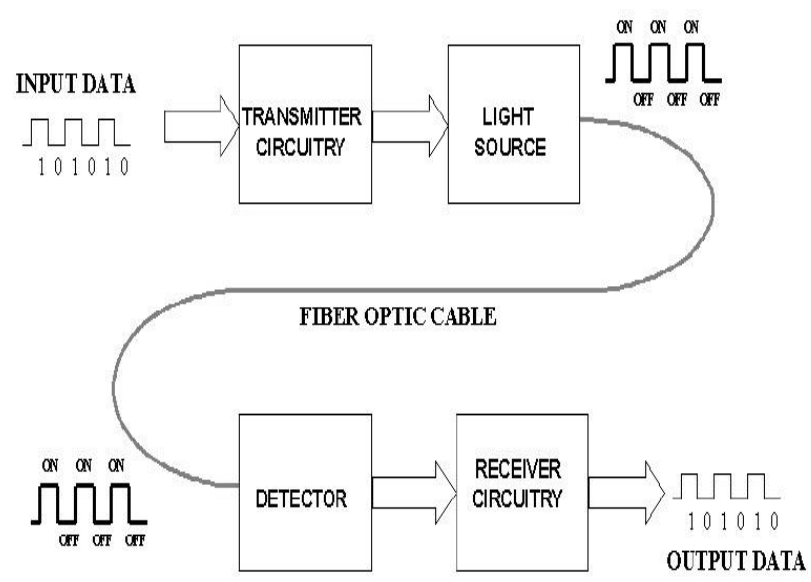

Fig. 1 Block Diagram of Fiber Optic System [1]

Various modulation formats are used at transmitter section, out of which Non Return to Zero (NRZ) and Return to Zero (RZ) are basic modulation formats. In logic circuit, logic clock is incorporated with which the output signal changes. When logic state does not change then there is no pulse transition at output, this is a condition of non-return to zero (NRZ). If logic state changes then a pulse or transmission edge occurs [11].Fig. 2 illustrates Optical Spectrum Analyzer of NRZ modulation. 


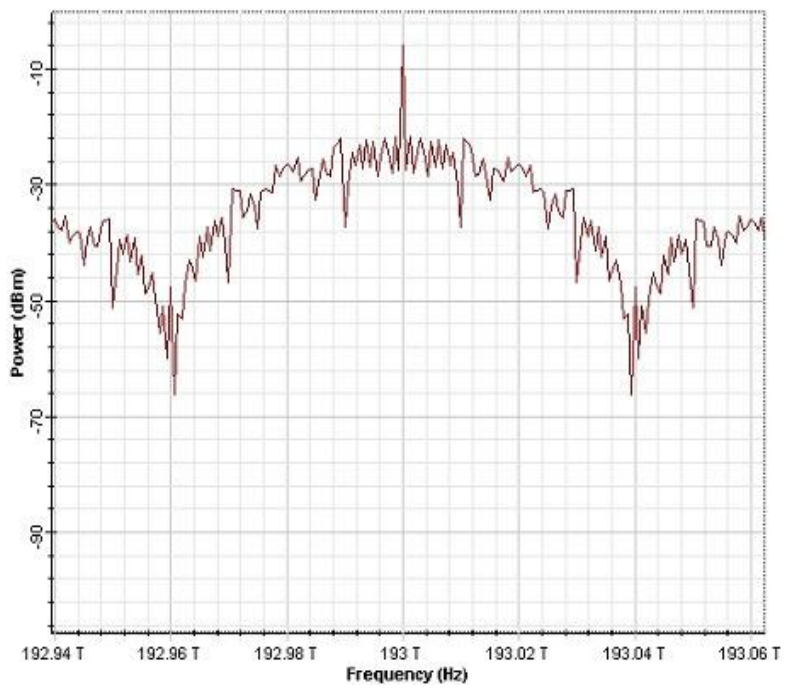

Fig. 2Optical spectrum analyzer of NRZ[11]

In RZ signals, logic pulse transition occurs at the state 'zero'. When logic state is 'one' then the pulse transition occurs half clock period. If logic state is 'zero' then no pulse occurs and the signal remains same [11].Fig. 3 illustrates Optical Spectrum Analyzer of RZ modulation.

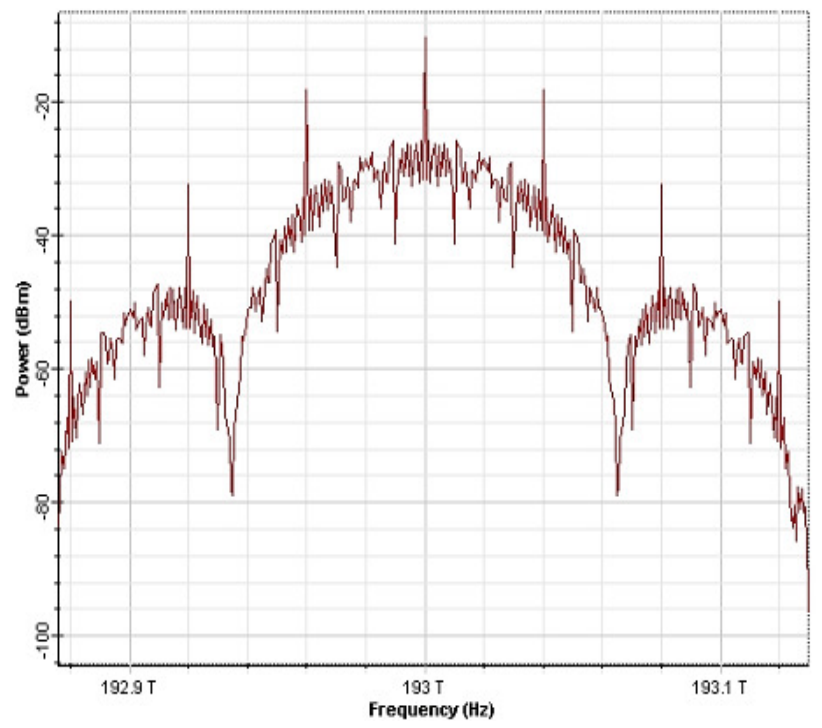

Fig. 3Optical spectrum analyzer of RZ[11]

Optical amplifiers are used extensively in fiber optic data communication links. EDFA improves signal to noise ratio in a link and amplify the signal at a wavelength of $1550 \mathrm{~nm}$ because here it shows minimum attenuation. In Semiconductor Optical Amplifier (SOA) medium is undoped. InGaAsP is used to provide optical amplification at a wavelength of 1.3 or $1.5 \mu \mathrm{m}$. Some other semiconductors are also used which can amplify the signal at different wavelength. Basically SOA is pumped with an electrical current. EDFA shows less amount of noise as compared to SOA and generally handle less amount of power. SOA is less expensive that's why use for local area networks. In RAMAN amplifiers, gain medium is undoped optical fiber. The wavelengths which experience optical gain are determined by the wavelength of optical pump, so Raman amplifier can be made to amplify a wavelength by proper selection of the pump wavelength.

\section{LITERATURE SURVEY}

D. Sharma, Y. K. Prajapati[2] have introduced about DWDM with different number of channel and different modulation scheme for achieving high data rates. BER and Q-fis observed by changing in number of channel, spacing and fiber length. The analysis has been performed with different modulation schemes.

S. K. Gill, G. Kaur[3] have considered 32 channels andsystem is analyzed for $125 \mathrm{~km}$ and $80 \mathrm{~km}$ distance using Dispersion Compensated Fiber (DCF)\&Fiber Bragg Grating(FBG). The performance of NRZ \& RZ modulation scheme has been observed and it is seen thatRZ gives better performance as compared to NRZ.

B.P. Patel, R. B. Patel[4] have introduced various modulation format of WDM with data rate $8 * 40$ Gbps. WDM system performancehas been analyzed by keeping input power in the range of -15 to $10 \mathrm{dbm}$ over a fixed distance of $300 \mathrm{~km}$. It is observed for different power levels and channel spacing, each modulation format have different performance.

N. V. Jaya, T. Kabilan[5] has discussed about the performance of DWDM systems. This paper is related to the performance of 48 channels DWDM using EDFA over long distance. In the simulation set up,32 channels are used for DWDM network.RZmodulation format is used to increase quality factor.

N.Rani [6] hasdemonstrated that performance of WDM systems depends upon modulation format used in the network. The proposed design of the system has maximum distance of $120 \mathrm{~km}$ at the power of $0-2 \mathrm{dbm}$ over bidirectional multi-mode fiber. The results of the designed system proved that Bidirectional 8 channel WDM-Passive Optical Network (PON)has enhanced performance with Modified Duo binary RZ (MDRZ) modulation format upto a distance of $120 \mathrm{~km}$, power $1 \mathrm{dBm}$ and channel spacing 200 $\mathrm{GHz}$, without using any amplifiers, DCF and repeaters.

V.Senthamizhselvan, R. Ramachandran, R. Rajasekar[7]have analyzed the performance of DWDM with NRZ and RZ data formats. Its transmitter section consists of 20 channels, input power $7 \mathrm{dBm}$, channel spacing $100 \mathrm{GHz}$, fiber length $100 \mathrm{~km}$. It has been observed that the RZ performs better than NRZ modulation.

Y. J. Wen, J. Mo, Y. Wang [8] have discussed about various schemes for advanced modulation formats in terms of configuration and cost. They have presented some configurations for optical data generation with better performance and reduction in components.

R. Mehra, V. Joshi[9] have introducedQ-f \& Eye diagram representation for RZ and NRZ modulation format. It is observed that NRZ modulation performs better than RZ at $1310 \mathrm{~nm}$ wavelength and at $1550 \mathrm{~nm} \mathrm{RZ}$ shows better result.

J. Singh, P. Gilawat, B. Shah[10] have simulated and analyzed $32 \times 40 \mathrm{Gbps}$ free space optical link that is of $1 \mathrm{~km}$ length and incorporates NRZ and RZ line coding. Here NRZ line coding has shown better performance. It has been observed that as the power increases, the BER decreases and Q-f increases.

S. Arya,

Joseph[11]have introduced
D.

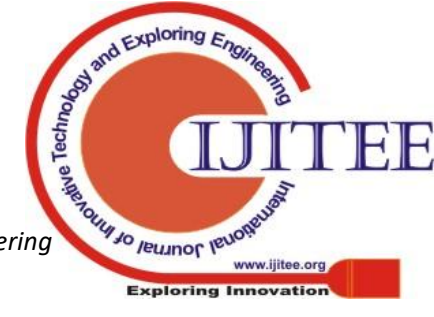


about the study the $\mathrm{L}$ band transmission for NRZ and RZ format at wavelength of $1610 \mathrm{~nm}$. It has been concluded that RZ format gives better results thanNRZ format.

Payal, S. Kumar, D. Sharma[12] have studied about DWDM system using EDFA in C-band and the performance of Return to Zero (RZ) and Non-Return to Zero (NRZ) modulation formats in an optical communication system is investigated. It is seen that as the fiber length increase the value of Q-f decreases and BER increases.

M. Sharma, N. Kaur [13] have analyzed 16 channel DWDM system using different modulation formats and compensation schemes for different bit rates. It is observed that at high speed, advanced modulation and dispersion compensation schemes give better results.

A.A. Khadir, B. F. Dhahir[14] have demonstrated the use of Optisystem software for modeling different design technologies of optical networks.P. Jain, K. Vashist, N. Gupta[15] have investigated about the performance of DWDM system for enhancing the capacity of the communication system through Hybrid Optical Amplifier. H. Ziqiang , L. Hongzuo , Z. Ting [16] have introduced about Erbium laser amplifier. This paper is related to transient effects of EDFA that are caused by the signal and pump power. The conclusions show that when the pump power is fixed, saturation time decreases, and input signal gain recovery time increases.

\section{SIMULATION MODEL}

The layout of design(DWDM_MOD) has been designed by OPTISYSTEM 16.0.Fig. 4 shows 56-channel DWDM network having RZ and NRZ modulation formats at 40 GB/s. The transmitter section consists of a 56-channel WDM transmitter with frequency and spacing of $1550 \mathrm{~nm}$ and $100 \mathrm{GHz}$ respectively. A 56- channel ideal multiplexer is also attached with WDM transmitter. Optical link has a length of $50 \mathrm{Km}$ of Single Mode Fiber (SMF), $10 \mathrm{Km}$ of Dispersion Compensated Fiber (DCF) and two optical amplifiers are used. The receiver consists of 56-channel WDM Demultiplexer, with an optical receiver and Bit Error Rate(BER) analyzer. The amplifiers are connected at output of SMF and DCF respectively. The combinations considered are EDFA post SMF and EDFA post DCF (EDFA+EDFA), SOA post SMF and SOA post DCF (SOA+SOA), and EDFA post SMF and SOA post DCF (EDFA + SOA). Hybrid optical amplifier configurations (SOA+SOA, EDFA+EDFA, EDFA+SOA)have been used to enhance the system performance. Simulation parameters are tabulated in Table I.

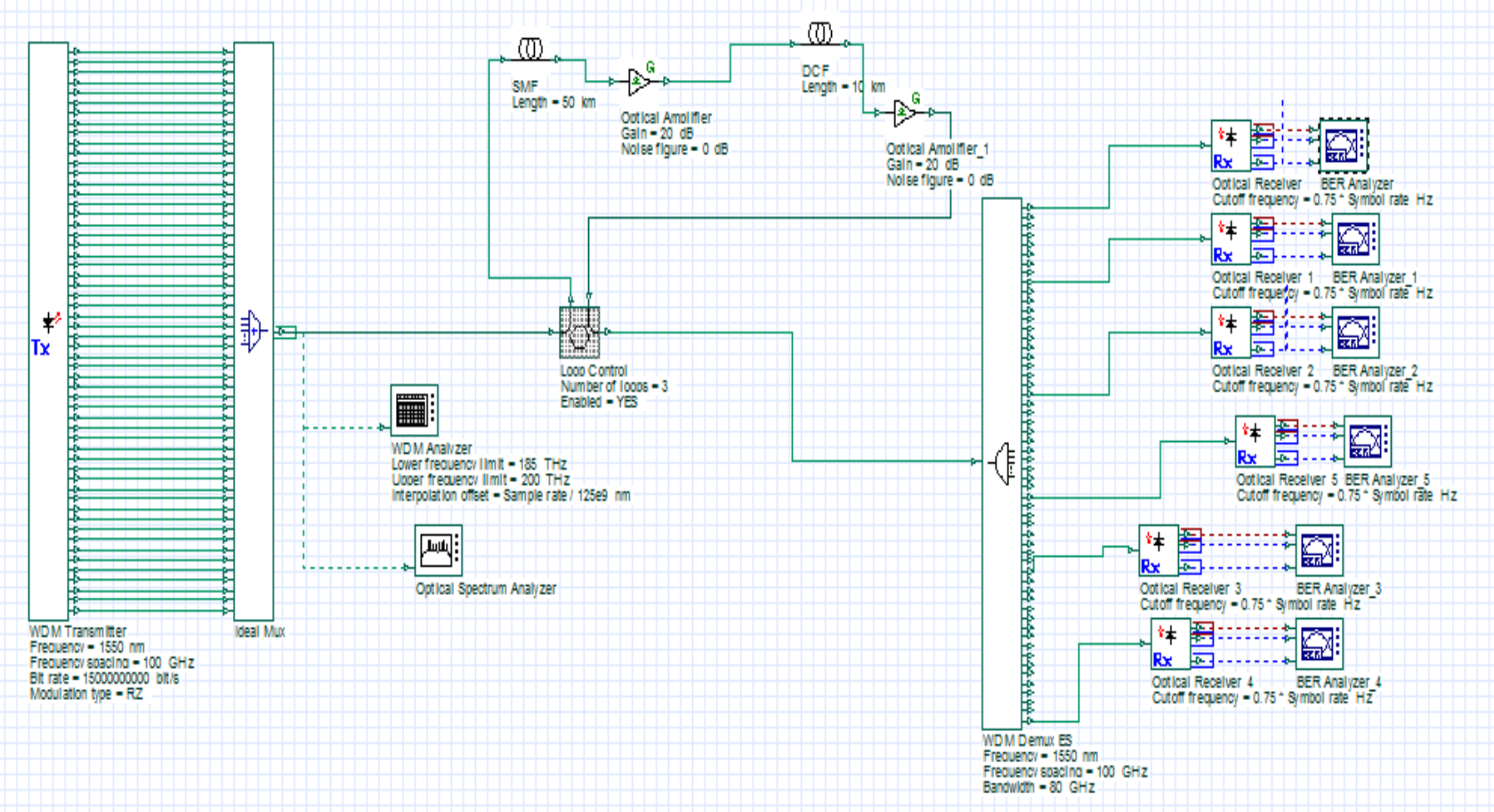

Fig. 4 Link Design: DWDM_MOD 
Applicability of Modulation Schemes for WDM High Speed Networks using Hybrid Optical Amplifiers

Table I. Simulation Parameters

\begin{tabular}{|l|l|}
\hline Parameter & Value \\
\hline Reference frequency & $1550 \mathrm{~nm}$ \\
\hline Frequency spacing & $100 \mathrm{GHZ}$ \\
\hline Optical fiber length & $240 \mathrm{~km}$ \\
\hline SMF length & $50 \mathrm{~km}$ \\
\hline DCF length & $10 \mathrm{~km}$ \\
\hline Optical amplifier gain & $20 \mathrm{db}$ \\
\hline Receiver cutoff frequency & $0.75 \mathrm{x} \mathrm{Bit} \mathrm{rate} \mathrm{Hz}$ \\
\hline Bit rate & $40000000000 \mathrm{bit} / \mathrm{s}$ \\
\hline Band width & $80 \mathrm{GHZ}$ \\
\hline Modulation format & $\mathrm{NRZ}$ \\
\hline Noise figure & $6 \mathrm{db}$ \\
\hline Power & $3.9999999564 \mathrm{dbm}$ \\
\hline
\end{tabular}

\section{RESULTS}

Eye diagram representation for RZ and NRZ modulation at various output analyzers are observed. Fig. 5 (a) - 5 (f)illustrates the output of BER analyzers depicting bit error rate at corresponding channels for NRZ modulation.Fig. 5 (a) - 5 (f) is for BER A, BER A1, BER A2, BER A3, BER A5 and BER A4 respectively (analyzers are depicted in Fig. 4). Quality Factoras observed at analyzers is $15.28,11.8379,12.4318,11.9485,11.8963$ and 15.165 respectively. The various analyzers are taken at randomly selected channels at output with even channel spacing. The link output parameters observed at these selected channels gives a range in which BER and Q-f varies at output from maximum to minimum values. The sequence of analyzers depicted in Fig. 5 is in accordance with the model shown in Fig. 4. The amplifiers in the simulation model are configured in hybrid combinations so as to further enhance the overall system performance.

\section{Analyzer(A) Eye Diagram}

\section{BER A}

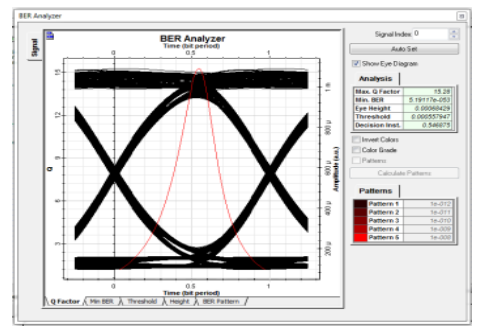

5(a)

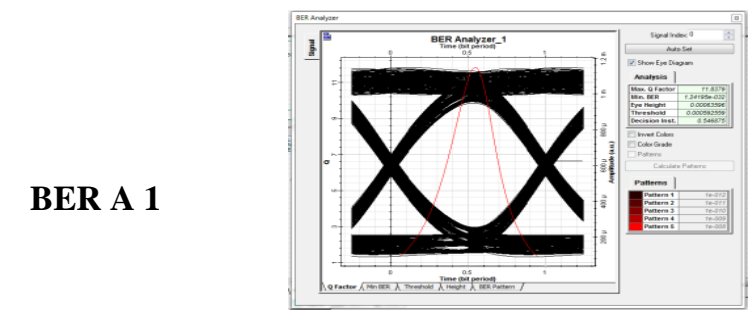

5(b)

\section{BER A 1}

BER A 2

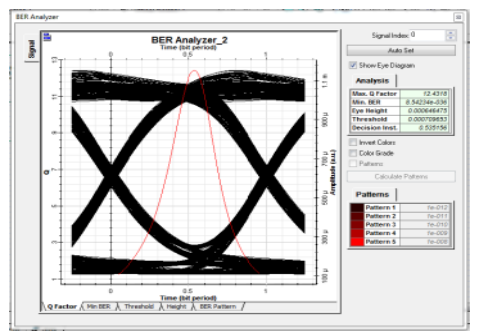

5(c)

BER A 3

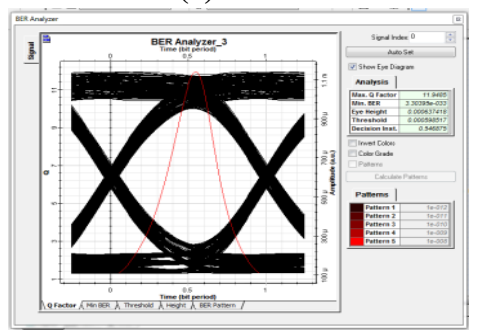

5(d)

BER A 5

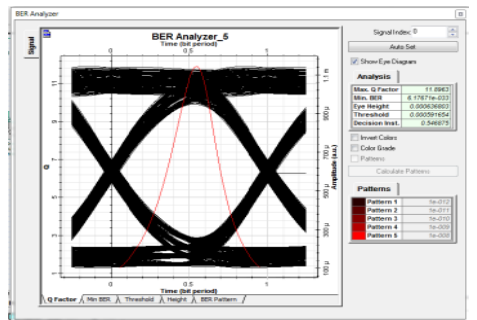

5(e)

BER A 4

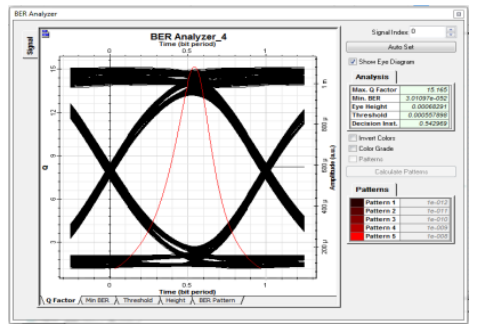

5(f)

Fig. 5 NRZ Modulation results

Fig. 6 (a) - 6 (f) illustrates the output of BER analyzers depicting bit error rate at corresponding channels for $R Z$ modulation. Fig. 6 (a) - 6 (f) is for BER A, BER A1, BER A2, BER A3, BER A5 and BER A4 respectively (analyzers are depicted in Fig. 4). Quality Factor is 14.3202, 9.90275, 9.24685, 9.99739, 9.70705 and 13.5993 and Bit Error Rate is $8.13588 \mathrm{e}-047,2.01182 \mathrm{e}-023,1.13782 \mathrm{e}-020,7.77134 \mathrm{e}-$ $024,1.39627 \mathrm{e}-022,2.00885 \mathrm{e}-042$ respectively. The quality factor has been enhanced with hybrid optical amplifier. Various hybrid configurations used are SOA+SOA, EDFA+EDFA, EDFA+SOA. System performance has been increased with application of these amplifiers with NRZ format. NRZ format has shown maximum improvement in quality factor with EDFA+EDFA hybrid configuration. 
Analyzer(A)

Eye Diagram

BER A

BER A 1

BER A 2

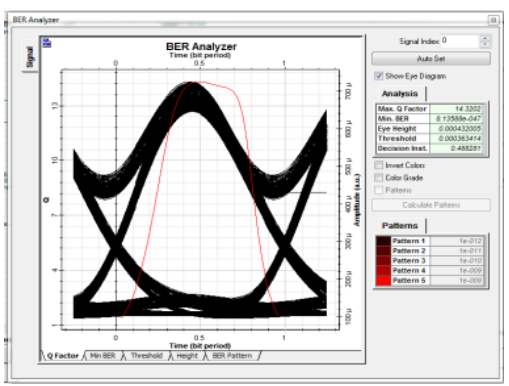

6 (a)

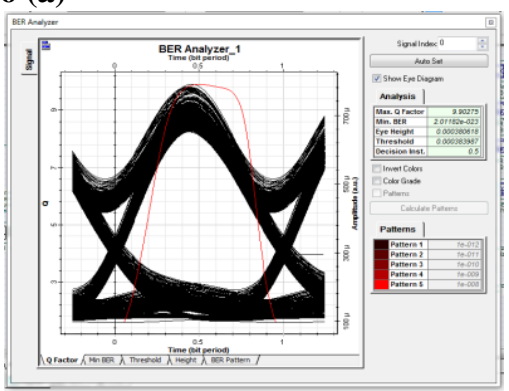

6 (b)

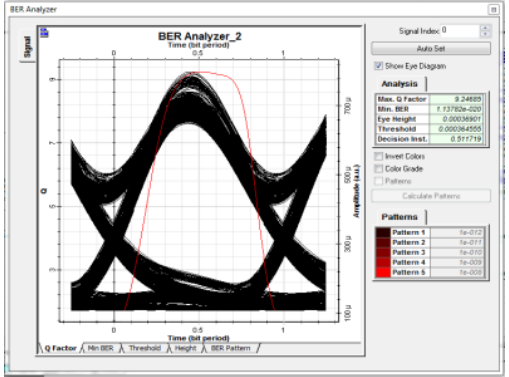

6 (c)

BER A 3

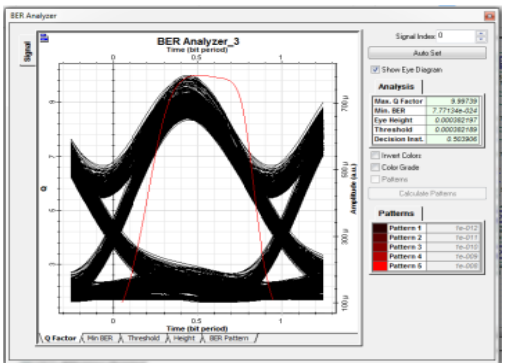

6 (d)

BERA 5

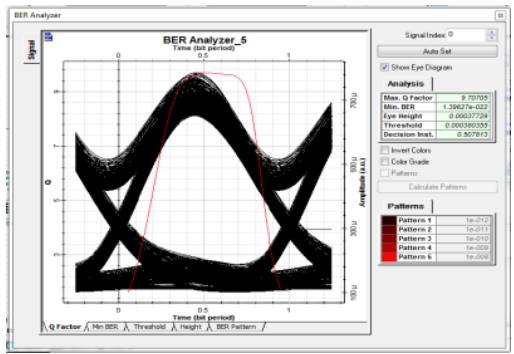

$6(\mathbf{e})$
BER A 4

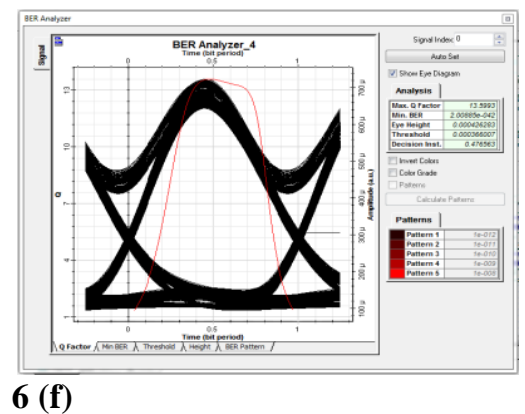

Fig. 6 RZ Modulation results

System output parameters for RZ and NRZ modulation are summarized in Table II.

Table II. Q-f and BER for RZ and NRZ Modulation

\begin{tabular}{|c|c|c|c|c|}
\hline & \multicolumn{2}{|l|}{ NRZ } & \multicolumn{2}{|l|}{$\mathrm{RZ}$} \\
\hline $\begin{array}{r}\text { A } \\
\text { nal- } \\
\text { yzer }\end{array}$ & Q-f & BER & Q- f & BER \\
\hline A & 15.28 & $\begin{array}{l}5.19117 \\
\text { e- } 053\end{array}$ & 14.3202 & $\begin{array}{l}8.13588 \\
\text { e- } 047\end{array}$ \\
\hline${ }_{1}{ }^{\mathrm{A}}$ & 11.8379 & $\begin{array}{l}1.24195 \\
\mathrm{e}-032\end{array}$ & 9.90275 & $\begin{array}{l}2.01182 \\
\mathrm{e}-023\end{array}$ \\
\hline $2^{\mathrm{A}}$ & 12.4318 & $\begin{array}{l}8.54234 \\
\text { e- } 036\end{array}$ & 9.24685 & $\begin{array}{l}1.13782 \\
\mathrm{e}-020\end{array}$ \\
\hline $3^{\mathrm{A}}$ & 11.9485 & $\begin{array}{l}3.30395 \\
\text { e- } 033\end{array}$ & 9.99739 & $\begin{array}{l}7.77134 \\
\mathrm{e}-024\end{array}$ \\
\hline $5^{\mathrm{A}}$ & 11.8963 & $\begin{array}{l}\begin{array}{c}6.17671 \\
\text { e- } 033\end{array} \\
\end{array}$ & 9.70705 & $\begin{array}{l}1.39627 \\
\mathrm{e}-022\end{array}$ \\
\hline $4^{\mathrm{A}}$ & 15.165 & $\begin{array}{l}3.01097 \\
\mathrm{e}-052\end{array}$ & 13.5993 & $\begin{array}{l}2.00885 \\
\mathrm{e}-042\end{array}$ \\
\hline
\end{tabular}

The link design DWDM_MODhas been compared with some of the approaches in literature. It is observed that proposed design has better performance in terms of Quality factor and BER.DWDM_MOD has Quality Factor of 15.28 with NRZ data format. Improvement has been observed over various approaches in literature like in [5] Q-f is 11.67 with NRZ data format and in [6] Q-f is 8.97. BER performance has also been improved.

The system performance has further been enhanced with hybrid optical amplifiers. EDFA+EDFA configuration has enhanced the Q-f of system to 46.528. This is highest as compared to SOA+SOA and EDFA+SOA configurations with Q-f of 46.314 and 46.372 respectively. 


\section{Applicability of Modulation Schemes for WDM High Speed Networks using Hybrid Optical Amplifiers}

\section{CONCLUSION}

The high speed network link has been designed in OPTISYSTEM 16.0. Simulation results show that NRZ modulation technique results in improved network performance in terms of Q-f and BER. Highest Q-f and minimum BER has been obtained using NRZ scheme. The network performance has been observed for $240 \mathrm{Km}$ fiber length. The design is further enhanced with hybrid optical amplifier configurations. The amplifiers are connected at output of SMF and DCF respectively. The combinations considered are EDFA+EDFA, SOA+SOA and EDFA+SOA. NRZ modulation format with EDFA+EDFA hybrid optical amplifier configuration has enhanced the Q-f of system to 46.528. This is highest as compared to $\mathrm{SOA}+\mathrm{SOA}$ and EDFA+SOA amplifier configurations having Q-f of 46.314 and 46.372 respectively. The design can be further extended for more dense multiplexing, higher transmission rate, narrow channel spacing and longer distance of transmission.

\section{REFERENCES}

[1] Available: accessed on 1 May 2019 at 8:00 PM ISThttps://www.fs.com/the-advantages-and-disadvantages-of-fiberoptic-transmission-aid-431.html

[2] D. Sharma, Y. K. Prajapati, "Performance analysis of DWDM system for different modulation schemes using variations in channel spacing",J opt commun netw, vol. 37, no. 4, 2016, pp. 401-413

[3] S. K. Gill, G. Kaur, "Performance evaluation of 32 channel DWDM system using dispersion compensation unit at different bit rates", International Research Journal Of Engineering And Technology, vol. 03, no. 06, June 2016, pp. 2436-2441.

[4] B.P. Patel, R. B. Patel, "Comparison of different modulation formats for 8 channel WDM optical network at 40 GBPS datarate with nonlinearity," IJARET, vol. 5, no. 2, Feb. 2014, pp. 37-51.

[5] N. V. Jaya, T. Kabilan, "Performance analysis of 48 channels dwdm system using edfa for long distance communication," GRD Journals, vol. 2 , no. 3 , Feb. 2017 , pp. 39-47.

[6] N. Rani, "Design and implementation of various advanced modulation format over 8-wdm bidirectional PON,',IJARIIT, vol. 3, no. 6, 2017, pp. 300-305

[7] V.Senthamizhselvan, R. Ramachandran, R. Rajasekar, "Performance analysis of DWDM based fiber optic communication with different modulation schemes and dispersion compensation fiber",IJRET, vol. 03, no. 03, March 2014, pp. 287-290.

[8] Y. J. Wen, J. Mo, Y. Wang, "Advanced data modulation techniques for WDM transmission”, IEEE Commun. Mag., vol.44, no. 8, Aug. 2006, pp. 58-65.

[9] R. Mehra, V. Joshi, "Effect on Q factor of fixed bit pattern, and encoding techniques in intensity modulated optical networks".IJCA (0975 - 8887),vol. 106, no. 13, Nov. 2014, pp. 42-45

[10] J. Singh,P.Gilawat, B. Shah, "Performance evaluation of $32 \times 40$ GBPS (1.28 TBPS) FSO link using RZ and NRZ line codes,'IJCA (0975 8887), vol. 85, no 4,Jan. 2014,pp. 32-36.

[11] S. Arya, D. Joseph, "A study performance of 1- band optical communication system for NRZ and RZ format,"Int J Phys Chem Math Sci, vol. 4, no. 1, 2015,pp. 9-13.

[12] Payal, S. Kumar, D. Sharma, "Performance Analysis of NRZ and RZ Modulation Schemes in Optical Fiber Link Using EDFA,'IJARCSSE, vol. 7, no. 8, Aug. 2017, pp. 161-168.

[13] M. Sharma, N. Kaur, "Analysis of DWDM System Using Different Modulation and Compensation Technique at Different Bit Rates," International Journal of Exploring Emerging Trends in Engineering, vol. 02, no. 04, 2015, pp. 219-223.

[14] A.A. Khadir, B. F. Dhahir, "Achieving optical fiber experiments using Optisystem”, IJCSMC, vol. 3, no. 6, 2014, pp. 42-53.
[15] P. Jain, K. Vashist , N. Gupta, “Comparison Study of Hybrid Optical Amplifier,” IJSRET, vol. 3, no. 9, Dec. 2014, pp. 1289-1292.

[16] H. Ziqiang , L. Hongzuo , Z. Ting , "Theoretical analysis on the transient characteristics of EDFA in optical fiber communication," JASEI ,vol.1, no.2, 2014, pp. 83-91.

\section{AUTHORS PROFILE}

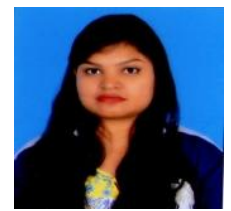

Shakshi is a second year M.Tech student in Electronics and Communication Engineering department at Deenbandhu Chhotu Ram University of Science and Technology, Murthal, Haryana. Her research area is optical communication and networking.

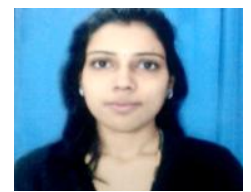

Himanshi Saini is Assistant Professor in Electronics and Communication Engineering department at Deenbandhu Chhotu Ram University of Science and Technology, Murthal, Haryana. She has above 50 publications in international/national journals and conferences. Her research area is optical networking and switching systems. 